\title{
Artificial Liver Support System for Fulminant Hepatic Failure as Bridge-use to Living Donor Liver Transplantation
}

\begin{abstract}
Key words: artificial liver support, living donor liver transplantation, fulminant hepatic failure
\end{abstract}

There are types of acute hepatic failure. One is viral, drug, or toxic acute hepatic failure without chronic liver disease. The other is "acute-on-chronic" hepatic failure; a metabolic event-induced acute hepatic failure with chronic liver disease. Wilsonian fulminant hepatic failure is a representative disease of the latter type. Both types indicate a different prognosis and treatment strategy, but, should be considered as a candidate of artificial and bioartificial support systems to attain recovery or liver transplantation. For these clinical events, artificial liver support systems have been employed for 20 years with the advances of hemodialysis, hemofiltration and hemoperfusion. From ten years ago, artificial liver support systems have progressed toward a combination of hemodialysis and hemodiabsorption. Recently, bioartificial liver support utilizing living hepatocytes was established (1). The recent advances in liver transplantation $(2,3)$ have achieved cure in approximately $75 \%$ of patients with acute hepatic failure.

In this editorial, I would like to summarize whether these artificial and bioartificial support systems may provide a beneficial bridge-use to transplantation for patients with acute hepatic failure.

\section{Clinical significance of plasma exchange (PE) and hemodialysis (HD)}

Supplementation of hepatic products, treatment of inflammation and transient improvement of impaired blood coagulation system can be obtained by PE with abundant fresh frozen plasma. Reduction of an organ disorder is also pursued with a decrease of serum bilirubin in this PE. On the other hand, the stability of circulatory dynamics, improvement of hepatic encephalopathy, and the removal of toxic substance are expected by HD. In fact, these are standard treatments for acute hepatic failure with encephalopathy at present.

Clinical results of artificial liver support systems for bridge-use to transplantation

To date, there are numerous reports concerning artificial and bioartificial supports system as bridge-use for the patients with severe liver disease to recovery or transplantation.
Kjaergard et al (4) selected and reviewed 12 randomized controlled studies utilizing artificial liver support systems (5 of BioLogic-DT, 2 of hemodiabsorption with albumin, 1 each of whole-blood exchange, charcoal hemoperfusion, plasma exchange with hemoperfusion, and 2 of bioartificial systems). Thus, 8 of the 12 studies used combined hemodialysis and hemodiabsorption.

It was based on stratified meta-analyses (5). They concluded that meta-analysis of 4 trials (6-9) indicated no significant effect on bridge-use to liver transplantation (Table 1), but there was a significant positive effect on hepatic encephalopathy in the other 8 trials. Furthermore, artificial support systems reduced mortality in acute-on-chronic liver failure compared with standard medical treatment. However, these systems did not appear to affect the mortality in acute liver failure.

Based on the results of this meta-analysis, should we conclude that these artificial liver supports are not necessary prior liver transplantation? Liver transplant for fulminant hepatic failure from cadaveric and living donor livers will be in the same condition (10). PE and HD will not demerit but will slightly improve encephalopathy, lack of hepatic synthesis, hemorrhagic tendency, and the unbalance of circulatory dynamics before liver transplantation. There is nothing disadvantageous in some removal by $\mathrm{HD}$, and some supply by $\mathrm{PE}$ for the predisposing condition of multiorgan failure (11) in fulminant hepatic failure. The problem is the numerous adverse events, such as infection and hemorrhagic tendency.

\section{Clinical significance of $P E$ and $H D$ for Wilsonian fulminant hepatic failure}

This report (12) describes that these artificial liver support systems can provide an additional benefit particularly for Wilsonian fulminant hepatic failure.

\section{See also p 967.}

In acute-on-chronic cases, this artificial liver support is extremely useful by contributing to the survival rate in randomized control studies. Wilsonian fulminant hepatic failure is based on the liver cirrhotic state of Wilson disease. Furthermore, the authors consider the benefit of the rapid removal of serum-free copper, in addition to liver support for acute hepatic failure. This greatly improves hemolytic anemia. This method is a logical therapy, and actually removes copper quickly before the effect of d-penicillamin appears. 
Table 1. Randomized Controlled Studies of Artificial Liver Support for Acute Hepatic Failure before Liver Transplantation

\begin{tabular}{lllll}
\hline \multicolumn{1}{c}{$\begin{array}{c}\text { Source } \\
\text { (Year) (Ref.) }\end{array}$} & $\begin{array}{c}\text { Intervention } \\
\text { Devices }\end{array}$ & $\begin{array}{c}\text { Type of } \\
\text { failure }\end{array}$ & $\begin{array}{c}\text { Efficacy } \\
\text { ALSS control }\end{array}$ \\
\hline O'Grady (1988) (6) & Charcoal-HP & Acute & $19 / 29$ & $20 / 33$ \\
Mazariegos (1997) (7) & BioLogic-DT & Acute & $1 / 5$ & $1 / 5$ \\
Kramer (1998) (8) & BioLogic-DT & AOC & $4 / 10$ & $4 / 10$ \\
Wilkinson (1998) (9) & BioLogic-DT & AOC & $3 / 5$ & $3 / 5$ \\
\hline
\end{tabular}

HP: hemoperfusion, Acute: acute hepatic failure, AOC: acute on chronic hepatic failure, ALSS: artificial liver support system.

Therefore, PE and CHDF for Wilsonian fulminant hepatic failure are the most superior liver support system and, I think they are indispensable as a treatment before liver transplant. In addition, in consideration of medical economics, this support system is more useful for living donor liver transplantation as a programming operation than cadaveric liver transplantation in such a donor shortage period with a long waiting time.

Takafumi ICHIDA Life Science Medical Center of Niigata University Hospital, Niigata University School of Medicine, 754 Asahimachi-Dori, 1, Niigata 951-8510

\section{References}

1) Strain AJ, Neuberger JM. A bioartificial liver-state of the art. Science 295: 1005-1009, 2002.

2) Matsunami H, Makuuchi M, Kawasaki S, et al. Living-related liver transplantation in fulminant hepatic failure. Lancet 340: 1411-1412, 1992.

3) Ichida T. Does brain damage (Hepatic encephalopathy) improve without any complication and sequellae after liver transplantation? Intern Med 39: 871-872, 2000.

4) Kjaergard LL, Liu J, Als-Nielsen B, Gluud C. Artificial and bioartificial support systems for acute and acute on chronic liver failure. A systematic review. JAMA 289: 217-222, 2003.

5) Moher D, Pham B, Jones A, et al. Does quality of reports of randomized trials affect estimates of intervention efficacy reported in metaanalysis? Lancet 352: 609-613, 1998.

6) O'Grady JG, Gimson AE, O'Brien CJ, Pucknell A, Hughes RD, Williams R. Controlled trials of charcoal hemoperfusion and prognostic factors in fulminant hepatic failure. Gastroenterology 94: 1186-1192, 1988.

7) Mazariegos GV, Ash SR, Patzer JF, et al. Preliminary results: randomized clinical trial of the BioLogic-DT in treatment of acute hepatic failure (AHF) with coma. Artif Organs 21: 529, 1997.

8) Kramer L, Gendo A, Madl C, et al. A controlled study of the BioLogicDT system in chronic hepatic encephalopathy. Hepatology 28: 401A, 1998.

9) Wilkinson AH, Ash SR, Nissenson AR, et al. Hemodiabsorption in treatment of hepatic failure. J Transpl Coord 8: 43-50, 1998.

10) Ichida $T$, Matsunami $H$, Kawasaki $S$, et al. Living related donor liver transplantation from adult to adult for primary biliary cirrhosis. Ann Intern Med 122: 275-276, 1995.

11) Riordan SM, Williams R. Acute liver failure: targeted artificial and hepatocyte-based support of liver regeneration and reversal of multiorgan failure, J Hepatol 32 (Suppl 1): 63-76, 2000.

12) Nagata $Y$, Uto $H$, Hasuike $S$, et al. Bridging use of plasma exchange and continuous hemodiafiltration before living donor liver transplantation in fulminant Wilson's disease. Intern Med 42: 967-970, 2003. 